\title{
AVALIAÇÃO DE CULTIVARES DE BANANEIRA EM SISTEMA DE CULTIVO CONVENCIONAL E ORGÂNICO ${ }^{1}$
}

\author{
LINDINÉIA RIOS RIBEIRO², LENALDO MUNIZ DE OLIVEIRA ${ }^{3}$, \\ SEBASTIÃO DE OLIVEIRA E SILVA4 ${ }^{4}$, ANA LÚCIA BORGES
}

RESUMO - A bananicultura apresenta importância social na geração de emprego e renda e como fonte alimentar para pessoas em todo o mundo. A crescente demanda por frutos de alta qualidade tem contribuído para a conversão de áreas manejadas convencionalmente para sistemas de manejo orgânico. Contudo, inexistem informações seguras referentes às vantagens de cada tipo de manejo, que possam subsidiar os agricultores nessa importante tomada de decisão. Assim, com o objetivo de comparar cultivares de bananeira, em manejo convencional e orgânico, foram avaliadas as características agronômicas e o estado nutricional das bananeiras, quantificando-se o teor de minerais nas folhas. Foram avaliadas as cultivares Caipira (AAA), Maravilha (AAAB), Pacovan Ken (AAAB), Prata-Anã (AAB), Thap Maeo (AAB) e Tropical (AAAB). Os dados foram submetidos à análise de variância, e as médias, comparadas pelos testes Scott Knott e Tukey, a 5\% de probabilidade de erro. O sistema de cultivo afetou os parâmetros agronômicos de bananeira, destacando-se a cultivar Thap Maeo, que apresentou superioridade para algumas características agronômicas, podendo ser facilmente utilizado em sistema orgânico de cultivo. As cultivares apresentaram diferenças agronômicas, independentemente do sistema de manejo adotado. $\mathrm{O}$ tipo de manejo afeta a maioria das variáveis, exceto para peso do cacho, peso total da penca, número de frutos por penca, despencamento e firmeza do fruto com casca.

Termos para indexação: Musa spp., sistema de manejo, produtividade, caracteres agronômicos.

\section{EVALUATION OF BANANA CULTIVARS IN CONVENTIONAL AND ORGANIC CROP SYSTEM}

\begin{abstract}
The banana has social importance in generating employment and income and as a food source for people around the world. However, the growing demand for high quality fruits have contributed to the conversion of conventionally managed areas for organic management systems. However, there is no reliable information concerning the advantages of each type of management. Thus, with the goal of improved banana cultivars compared to conventional and organic agronomic traits were evaluated cultivar Caipira (AAA), Maravilha (AAAB), Ken Pacovan (AAAB), Prata Anã (AAB), Thap Maeo (AAB) and Tropical (AAAB) grown in the two systems. In addition to agronomic traits, it was assessed the production and productivity and nutritional status of banana, quantifying the concentration of minerals in samples taken from the third leaf. Data were subjected to analysis of variance and means were compared by the Scott Knott test and Tukey test at $5 \%$ probability. The cropping system affects the agronomic parameters of banana, especially the cultivar Thap Maeo that had good agronomic characteristics and can be easily used in organic system. Agronomic cultivars differ, independent of the management system adopted.
\end{abstract}

Index terms: Musa spp., banana, management system, productivity, agronomic traits.

\footnotetext{
${ }^{1}$ (Trabalho 236-12). Recebido em: 28-08-2012. Aceito para publicação em: 07-06-2013. Trabalho desenvolvido como parte da dissertação de Mestrado do primeiro autor no Programa de Pós-Graduação em Recursos Genéticos Vegetais da Universidade Estadual de Feira de Santana.

${ }^{2}$ Pós-Graduanda em Recursos Genéticos Vegetais da Universidade Estadual de Feira de Santana. E-mail: neiarios@hotmail.com ${ }^{3}$ Doutor em Fisiologia Vegetal, Departamento de Ciências Biológicas da Universidade Estadual de Feira de Santana - UEFS, Av. Transnordestina, s/n, 44036-900, Feira de Santana-BA, Brasil - E-mail: lenaldo@uefs.br

${ }^{4}$ Doutor em Melhoramento Genético de Plantas, Universidade Federal do Recôncavo da Bahia, Rua Rui Barbosa, 710 - Centro, Cruz das Almas- BA, Brasil - E-mail: ssilva3000@gmail.com.br

${ }^{5}$ Doutora em Solo e Nutrição de Plantas, Embrapa Mandioca e Fruticultura, Rua Embrapa s/n. Cruz das Almas - BA, Brasil - E-mail: analucia@cnpmf.embrapa.br
} 


\section{INTRODUÇÃO}

A banana (Musa spp.) é uma das frutas mais produzidas e consumidas em todo o mundo devido ao seu valor nutricional. Ocupa o quarto lugar no ranking das fruteiras comerciais (DANTAS et al., 2011). Apesar de ser uma das commodities de grande interesse alimentar, sua produção é realizada, em quase sua totalidade, com uso de insumos químicos em sistema de manejo convencional; no entanto, o mercado tem demandado de forma crescente alimentos sem uso de agrotóxicos (LICHTEMBERG, L.A., et al.)

A bananicultura brasileira apresentou uma área cultivada correspondente a 511.000 hectares em 2009, manejados de forma convencional, porém áreas sob manejo orgânico estão crescendo consideravelmente em todo o mundo (TERRAZZAN et al., 2009), já representando mais de 1,5 milhão de hectares. O Brasil ocupa a oitava posição com 800.000 hectares, com uma estimativa de que $0,9 \%$ de área cultivada com banana está sob cultivo orgânico, percentual que cresce a cada ano (TERRAZZAN et al., 2009). Essa crescente demanda por alimentos orgânicos ocorre devido, principalmente, à preocupação dos consumidores com a segurança alimentar, em virtude dos altos níveis de agrotóxicos presentes em algumas culturas, que são facilmente absorvidos pelo organismo, podendo causar danos que vão desde leves intoxicações a graves problemas de saúde. Em pesquisa realizada pela ANVISA em 2009 (Agência de Vigilância Sanitária), e atualmente pelo MAPA (Ministério da Agricultura Pecuária e Abastecimento), foram detectados altos níveis de produtos químicos em frutas e hortaliças, acima do limite máximo de tolerância para a ingestão. Em análise de 17 diferentes culturas, realizada pela ANVISA em 2008, a banana chegou a apresentar índice de 1,53\% de contaminação por agrotóxicos.

Aliado ao problema de saúde, o sistema de cultivo convencional tem criado sérios problemas ecológicos, como a contaminação do solo e do lençol freático, interferindo na biologia dos animais e insetos que habitam esses ecossistemas. Os produtos químicos podem tornar as pragas mais resistentes, $\mathrm{o}$ que ocasiona o aumento crescente da dosagem do produto para um controle mais efetivo.

O melhoramento genético tem contribuído para a redução desses danos, com a geração de cultivares resistentes às principais pragas e doenças. Porém, a quantidade de tempo demandada para a produção de uma cultivar promissora é relativamente longo e, muitas vezes, não atende à demanda dos consumidores em relação à análise sensorial. Outra alternativa que tem sido adotada para minimizar esses problemas é a adoção do sistema integrado de controle das pragas e doenças, contudo essas medidas têm sido pouco efetivas quando comparadas com o aumento da demanda por alimentos sem uso de agrotóxicos, o qual é requerido em todo o mundo. Mais recentemente, a agricultura orgânica tem sido proposta como alternativa para a produção de bananas sem uso de agrotóxicos.

A agricultura orgânica é baseada em um sistema holístico, compatibilizando a produção de alimentos sem a utilização de insumos químicos na lavoura, aliado ao baixo custo de produção (AZADI; HO, 2010). Esse sistema de cultivo tem como princípio básico o aumento da diversidade biológica, para atingir um sistema natural considerado ideal para o cultivo de espécies, possibilitando, ao mesmo tempo, cumprir o papel social, econômico e ambiental, diferentemente do sistema convencional, que desequilibra o ecossistema, tendo a produtividade como foco principal.

Devido a esse novo apelo, aliado às melhores remunerações obtidas pelos produtos orgânicos, muitos produtores têm migrando do sistema de produção convencional para o orgânico. Contudo, ainda são poucas as informações técnicas a respeito das vantagens e desvantagens da adoção desse sistema. Diante disso, este trabalho teve como objetivo o estudo comparativo de caracteres agronômicos de cultivares de bananeira em sistema de cultivo convencional e orgânico, buscando-se subsidiar os produtores nessa importante tomada de decisão.

\section{MATERIAL E MÉTODOS}

Foram avaliadas as cultivares de bananeira Caipira (AAA), Maravilha (AAAB), Pacovan Ken (AAAB), Prata-Anã (AAB), Thap Maeo (AAB) e Tropical (AAAB) (Tabela 1) em experimentos instalados na Embrapa Mandioca e Fruticultura, em Cruz das Almas - BA, situados à latitude -12 $2^{\circ} 40^{\prime} 12^{\prime}$ " e longitude $-39^{\circ} 06^{\prime} 07^{\prime}$, em manejo convencional e orgânico. O experimento foi conduzido em Latossolo Vermelho-Amarelo, distrófico a moderado, textura francoargilosa, com pluviosidade média anual de $1.200 \mathrm{~mm}$ e umidade relativa de $80 \%$. As avaliações foram realizadas no período de 2009 a 2011.

As bananeiras foram adubadas no terceiro ciclo da cultura, de acordo com a análise química do solo, que apresentou as seguintes características na camada de 0-20 cm: a) sistema convencional - $\mathrm{Ca}$ $=2,5 \mathrm{cmolc} / \mathrm{dm}^{3} ; \mathrm{Mg}=1,00 \mathrm{cmolc} / \mathrm{dm}^{3} ; \mathrm{Al}=0,0$ $\mathrm{cmolc} / \mathrm{dm}^{3} ; \mathrm{Na}=0,15 \mathrm{cmolc} / \mathrm{DM}^{3} ; \mathrm{H}+\mathrm{Al}=1,54$ $\mathrm{cmolc} / \mathrm{dm}^{3} ; \mathrm{S}=3,79 \mathrm{cmolc} / \mathrm{dm}^{3} ; \mathrm{CTC}=5,33 \mathrm{cmolc} /$ $\mathrm{dm}^{3} ; \mathrm{V}(\%)=71$ e $\left.\mathrm{MO}=12,31 \mathrm{~g} / \mathrm{kg} ; \mathrm{b}\right)$ em sistema 
orgânico $-\mathrm{pH}\left(\mathrm{H}_{2} \mathrm{O}\right)=4,9 ; \mathrm{Ca}=2,5 \mathrm{cmolc} / \mathrm{dm}^{3} ; \mathrm{Mg}$ $=0,3 \mathrm{cmolc} / \mathrm{dm}^{3} ; \mathrm{S}=3,1879 \mathrm{cmolc} / \mathrm{dm}^{3}, \mathrm{CTC}=$ $7,91 \mathrm{cmolc} / \mathrm{dm} 3, \mathrm{~V}=40 \%$ e $\mathrm{MO}=7,5 \mathrm{~g} / \mathrm{kg}$.

Com base nos resultados acima, procedeuse à fertilização do solo com ureia $\left(100 \mathrm{~kg} \mathrm{ha}^{-1}\right)$, superfosfato simples $\left(280 \mathrm{~kg} \mathrm{ha}^{-1}\right)$ e cloreto de potássio $\left(540 \mathrm{~kg} \mathrm{ha}^{-1}\right)$ no sistema convencional e no sistema de cultivo orgânico, com composto orgânico (10 litros por cova) e fosfato natural (1 $\mathrm{kg}$ por cova) no plantio e composto orgânico $(2,5$ litros por planta) em cobertura e a cada seis meses de cultivo. No sistema orgânico, o espaço entre as fileiras de planta foi ocupado com cobertura viva, composta por amendoim forrageiro (Arachis pintoi) e feijão-de-porco (Canarvalia ensiformis). Em ambos os sistemas de cultivo, adotou-se o espaçamento de fileira dupla $(4,0 \mathrm{~m} \times 2,0 \mathrm{~m} \times 2,0 \mathrm{~m})$, procedendose irrigação complementar nos meses de setembro a janeiro dos respectivos anos, e não se utilizaram defensivos agrícolas para o controle de pragas.

As avaliações agronômicas foram realizadas no período do florescimento e da colheita do cacho, sendo considerados: a) altura da planta (APL), medida realizada no momento de emissão do cacho com auxílio de uma régua posicionada desde o nível do solo até o ponto de saída do engaço; b) diâmetro do pseudocaule (DIA), medida realizada no momento da emissão do cacho com auxílio de um paquímetro de madeira posicionado a $30 \mathrm{~cm}$ do solo; c) número de folhas presente na floração (NFVF), por contagem do número de folhas vivas presentes nas plantas na época de florescimento e da colheita (NFVC), considerando como funcional a folha que apresentava $50 \%$ a mais do limbo verde; e) ciclo da planta, por contagem dos dias do plantio à emissão do cacho (DPE); do plantio à colheita (DPC) e do plantio ao florescimento (DFC); f) presença de Sigatoka-amarela (SIA), avaliada no período de emissão do cacho e no momento da colheita. Para as características de produtividade, foram consideradas: a) peso total do cacho (PCA); b) peso total das pencas (PTP); c) número de frutos por cacho (NFP); d) número de pencas (NPE); e) comprimento médio do fruto na segunda penca (CMF); f) diâmetro médio do fruto na segunda penca (DMF); g) despencamento do fruto (DESP), e h) firmeza dos frutos com a casca (FCC) sem a casca (FSC). Para avaliar a resistência ao despencamento, foi utilizado o despencador mecanizado com os resultados expressos em libras, enquanto a firmeza do fruto com casca e sem casca foi realizada com um nanômetro de pressão posicionado no centro do fruto maduro (grau 6 da escala de Von Loseveck), com valores expressos em Newton (N). Além das variáveis agronômicas, quantificaram-se os teores de nutrientes nas folhas de bananeiras cultivadas em manejo orgânico e convencional. As folhas foram coletadas no momento da emissão da inflorescência, coletando-se a terceira folha, a contar do ápice da planta, quando as três brácteas masculinas se encontravam abertas e nenhuma bráctea feminina nesse estado. Utilizouse apenas a parte mediana da folha sem considerar a nervura principal, levando em seguida à estufa a $70^{\circ} \mathrm{C}$ para secagem até atingir massa constante. As amostras foram encaminhadas ao laboratório de Nutrição Mineral de Plantas para a determinação dos teores de macronutrientes (N, P, K, Ca, Mg e $\mathrm{S})$ e micronutrientes (B, Cu, Fe, Mn e $\mathrm{Zn}$ ), segundo metodologia proposta por Raij et al. (1997).

Foi adotado o delineamento inteiramente casualisado, com 10 repetições por tratamento e por cultivar para as características agronômicas, e três repetições para as características nutricionais. Os dados foram submetidos à análise de variância (ANOVA), e as médias, submetidas ao teste Scott Knott para comparar as cultivares, e Tukey para comparar os sistemas de cultivo, ambos a $5 \%$ de probabilidade de erro.

\section{RESULTADOS E DISCUSSÃO}

As características agronômicas da bananeira, segundo a análise estatística, apresentaram diferenças significativas em relação ao tipo de manejo utilizado para algumas variáveis. Para as cultivares, dentro dos sistemas de manejo, também se verificaram diferenças estatísticas para a maioria dos parâmetros avaliados, com exceção apenas para dias do florescimento à colheita. Para a interação dos fatores, verificou-se efeito significativo apenas para os caracteres número de folhas vivas no florescimento e na colheita, dias do plantio ao florescimento, do florescimento à colheita, firmeza do fruto sem casca e firmeza do fruto com casca e comprimento do fruto.

Verificou-se que a variável altura de planta se mostrou diferente em relação ao manejo adotado, com plantas de porte significativamente maior no sistema de manejo orgânico para todas as cultivares, à exceção da Prata-Anã (Tabela 2). Dentro de cada sistema de manejo, também se detectaram diferenças entre as cultivares para essa característica, obtendo-se maiores alturas na cultivar Pacovan Ken, com 366,58cm em sistema convencional; e em sistema orgânico, a Pacovan Ken, juntamente com a Thap Maeo apresentaram as maiores médias, com $402,76 \mathrm{~cm}$ e $381,61 \mathrm{~cm}$, respectivamente, não diferindo estatisticamente entre si. Oliveira et al. (2008), avaliando genótipos de bananeira, verificaram altura média de 4,08 
m para Pacovan Ken, enquanto Ramos et al. (2009) observaram altura média de 2,50 $\mathrm{m}$ para a cultivar Thap Maeo, valor bem abaixo do encontrado neste trabalho em sistema de cultivo orgânico. Gonçalves et al. (2008) afirmam que a altura de planta é um descritor fitotécnico e de melhoramento, e está ligada aos aspectos de densidade de plantio, produção e manejo da cultura. Já Santos et al. (2006) salientam que, em uma cultivar comercial, é indesejável que a bananeira expresse valores muito elevados para a altura de planta.

Em sistema orgânico, verificaram-se as maiores médias para o diâmetro do pseudocaule em relação ao cultivo convencional, nas cultivares Pacovan Ken, Thap Maeo e Tropical. Os menores diâmetros do pseudocaule foram verificados na cultivar Caipira e Thap Maeo, independentemente do sistema de cultivo (Tabela 2). Esta característica assume importância no melhoramento genético da bananeira, uma vez que está relacionada ao vigor da planta, além de refletir na capacidade de sustentação do cacho e na suscetibilidade ao tombamento.

O sistema de cultivo orgânico também possibilitou maiores valores para o número de folhas vivas no florescimento em relação ao manejo convencional, em que as cultivares Maravilha, Pacovan Ken e Thap Maeo apresentaram as maiores médias (Tabela 2). Dentro desse sistema, a cultivar Thap Maeo alcançou a maior média, enquanto os menores valores foram obtidos nas cultivares Caipira, Maravilha, Prata-Anã e Tropical (Tabela 2). Já no sistema convencional, destacaram-se as cultivares Prata-Anã, Pacovan Ken, Tropical e Thap Maeo com maior número de folhas vivas no florescimento (Tabela 2). Os maiores valores obtidos no sistema orgânico, para esse caráter, podem estar relacionados à presença de leguminosa nas entrelinhas, contribuindo para a maior disponibilização de nitrogênio para a emissão de folhas nas bananeiras.

O maior número de folhas no momento da colheita do cacho foi obtido pela cultivar Thap Maeo, tanto em sistema de cultivo convencional quanto no orgânico, embora, neste último, não tenha diferido estatisticamente das cultivares Caipira e Maravilha (Tabela 2). Comparando-se os sistemas de cultivo, verificou-se superioridade estatística do sistema orgânico apenas na cultivar Caipira.

Em relação aos parâmetros de precocidade, para o período compreendido entre o plantio e o florescimento, verificou-se efeito do sistema de cultivo apenas para a cultivar Maravilha, com maior precocidade no sistema convencional de cultivo (Tabela 3). Dentro dos sistemas de cultivo, as cultivares mais precoces foram Thap Maeo e Caipira, em sistema convencional, e Caipira, Pacovan Ken, Prata-Anã e Thap Maeo, em sistema orgânico. Por outro lado, considerando o número de dias do plantio à colheita, verificou-se maior precocidade em sistema de cultivo orgânico para as cultivares Caipira e Thap Maeo (Tabela 3). As cultivares que apresentam os menores intervalos entre o florescimento e a colheita têm a vantagem de menor permanência dos frutos em campo, ou seja, menores as chances de ocorrerem injúrias aos frutos, além do retorno econômico ser mais rápido. Para Santos et al. (2006), a redução do número de dias necessários para a emissão do cacho representa a antecipação do investimento aplicado e menor tempo de exposição do cacho a agentes causadores de danos, o que resulta em menos uso de defensivos agrícolas. O caráter ciclo da planta, da floração à colheita, é dependente das condições climáticas da região, como temperatura, umidade e precipitação, para o bom desenvolvimento da planta. Contudo, de modo geral, os resultados obtidos neste trabalho apontam para maior precocidade em algumas cultivares conduzidas de forma orgânica.

Para as características de produtividade das bananeiras, verificam-se diferenças significativas para o comprimento e o diâmetro do fruto (Tabela 4). O sistema orgânico possibilitou maior comprimento médio do fruto para as cultivares Pacovan Ken e Thap Maeo e maior diâmetro médio do fruto na cultivar Thap Maeo (Tabela 4).

Para a produção estimada em peso total dos cachos, verificou-se a superioridade das cultivares Maravilha e Thap Maeo, em ambos os sistemas de cultivo, enquanto para o peso total das pencas verificou-se maior valor para as cultivares Maravilha e Thap Maeo apenas no manejo convencional; enquanto no manejo orgânico os maiores valores foram obtidos nas cultivares Thap Maeo e Tropical (Tabela 4). Pode-se inferir que as diferenças obtidas entre as cultivares certamente são decorrentes de aspectos inerentes à genética das próprias cultivares, considerando-se que são expressas mesmo em condições edafoclimáticas diferentes.

A cultivar Thap Maeo apresentou o maior número de pencas por cacho em ambos os sistemas de cultivo (Tabela 4). Da mesma forma, a cultivar Thap Maeo apresentou o maior número de frutos por cacho, não sendo detectadas diferenças em relação ao sistema de cultivo. Os valores obtidos para o parâmetro número de frutos por cacho, para a cultivar Thap Maeo, são próximos dos encontrados por Santos et al. (2006), que obtiveram média de 220 frutos por cacho. O caráter número de pencas como de grande interesse para o produtor é fundamental para o melhoramento genético da bananeira, uma vez 
que a penca constitui-se na unidade comercial dessa cultura e está associado a outros parâmetros, como o comprimento e o diâmetro do fruto.

O comprimento e o diâmetro médios dos frutos também variaram em função das cultivares dentro de cada sistema de manejo. A cultivar Maravilha apresentou o maior comprimento de fruto no sistema convencional, enquanto as cultivares Pacovan Ken e Maravilha apresentaram frutos com maior comprimento no sistema orgânico, o que indica uma clara interação entre cultivar x sistema de manejo. Contudo, independentemente do sistema de cultivo, as cultivar Maravilha, Pacovan Ken e Tropical apresentaram os maiores valores para esse parâmetro (Tabela 5). Para o diâmetro médio do fruto verificou-se efeito do sistema de cultivo apenas para a cultivar Thap Maeo, com maior diâmetro no sistema orgânico (Tabela 5). Na comparação entre as cultivares, verificou-se que a cultivar Maravilha apresentou o maior diâmetro em sistema convencional, não sendo detectadas diferenças estatísticas entre as cultivares no sistema orgânico de cultivo.

Não se detectaram diferenças significativas para o despencamento do fruto entre os sistemas de manejo avaliados. De modo semelhante, não foi possível a distinção entre as cultivares, dentro do sistema orgânico, para esse caráter. Por outro lado, no sistema convencional, obteve-se maior resistência ao despencamento na cultivar Thap Maeo (Tabela 6). A resistência ao despencamento é determinada por fatores genéticos, exposição a uma atmosfera modificada e, principalmente, altas temperaturas e deficiência nutricional sendo uma importante variável para a produção do fruto, apresentando importância para o melhoramento genético dessa cultura (SANTOS et al., 2006).

Para a firmeza do fruto com a casca, também não se verificou efeito do sistema de cultivo, havendo distinção entre as cultivares apenas no sistema convencional, em que se verificou maior firmeza nos frutos das cultivares Maravilha, seguida da Caipira (Tabela 6). Em relação à firmeza do fruto sem casca, verificou-se grande variação dentro das cultivares e entre os sistemas de cultivo (Tabela 6), em que o sistema orgânico proporcionou frutos com polpa mais firme nas cultivares Caipira, Thap Maeo e Tropical. A maior uniformidade para os parâmetros de resistência ao despencamento e firmeza dos frutos com casca, em sistema orgânico, pode estar relacionada à maior disponibilidade de água e nutrientes nesse tipo de cultivo, efeito indireto da fertilização orgânica e da cobertura viva do solo.

Em relação à doença sigatoka-amarela, apenas duas cultivares apresentaram sintomas visíveis, tanto em sistema convencional quanto no orgânico, Prata Anã (98\% e 90\% das plantas afetadas, respectivamente) e Maravilha (92\% e $80 \%$ das plantas afetadas, respectivamente). As demais cultivares mostraram-se resistentes a essa doença em ambos os sistemas de cultivo.

Avaliando o estado nutricional das folhas, verificou-se variação significativa em algumas cultivares entre os resultados obtidos nos sistemas de cultivo (convencional ou orgânico) para a maioria dos nutrientes avaliados. $\mathrm{O}$ teor de nitrogênio não diferiu estatisticamente entre as cultivares dentro do sistema convencional. No entanto, em sistema orgânico, os maiores teores médios de $\mathrm{N}$ foram encontrados nas cultivares Caipira e Prata-Anã (Tabela 7). Para os teores de fósforo e enxofre em sistema convencional, não houve diferenças entre as cultivares. Por outro lado, houve diferença em relação ao tipo de manejo adotado, sobressaindo-se melhor no sistema convencional de cultivo. Tanto nos teores de fósforo como nos de enxofre em sistema orgânico, as maiores médias foram observadas na cultivar Caipira, que diferiu estatisticamente das demais cultivares (Tabela 7).

Damatto Júnior et al. (2006) trabalharam com diferentes doses de composto orgânico em 'Prata-Ana' e obtiveram teores foliares de $\mathrm{N}$ e $\mathrm{P}$ acima da faixa de suficiência. No presente trabalho, os teores de potássio nas folhas, no momento do florescimento, variaram de $25,16 \mathrm{~g} \mathrm{~kg}^{-1}$ para a cultivar Caipira a $30,36 \mathrm{~g} \mathrm{~kg}^{-1}$ para a cultivar Tropical, dentro do manejo convencional. Já no sistema orgânico, esse valor foi de 21,43 $\mathrm{g} \mathrm{kg}^{-1}$ para a cultivar Thap Maeo e $30,90 \mathrm{~g} \mathrm{~kg}^{-1}$ para a cultivar Caipira. Fatores internos, como cultivar, estádio fenológico das plantas, posição e porção das folhas, e fatores externos, como clima, solo e parasitismo devem ser levados em consideração em relação a uma adequada diagnose foliar, em que os teores foliares variam em relação ao tipo de cultivar.

Para os teores de macroelementos $(\mathrm{S}, \mathrm{Mg}$, $\mathrm{K}, \mathrm{Ca}$ ), verificaram-se maiores médias em folhas de plantas cultivadas em manejo convencional, devido provavelmente à composição em maior quantidade desse elementos na adubação química (Tabela 7). As plantas podem ter encontrado esses elementos disponíveis no solo, o que favoreceu sua absorção pela planta. Houve variação entre as cultivares dentro de cada sistema de cultivo convencional e orgânico, o que pode ser explicado pela genética de cada cultivar.

O maior teor foliar de cálcio foi encontrado no sistema convencional para as cultivares Caipira, Maravilha, Prata-Anã e Thap Maeo (Tabela 7), 
diferindo estatisticamente entre as demais cultivares (Tropical e Pacovan Ken), que apresentaram os menores teores de cálcio em ambos os sistemas de cultivo avaliados; contudo, seus teores médios foliares de Ca foram superiores ao padrão estabelecido para a cultura $\left(3,0 \mathrm{~g} \mathrm{~kg}^{-1}\right)$.

Já para os teores de magnésio, as cultivares Maravilha (3,90 $\left.\mathrm{g} \mathrm{kg}^{-1}\right)$, Caipira $\left(3,36 \mathrm{~g} \mathrm{~kg}^{-1}\right) \mathrm{e}$ Tropical $\left(3,30 \mathrm{~g} \mathrm{~kg}^{-1}\right)$ sobressaíram-se quando plantadas em sistema convencional, diferindo estatisticamente das mesmas cultivares quando em sistema de manejo orgânico, apresentando valores de $3,23 \mathrm{~g} \mathrm{~kg}^{-1} ; 2,40 \mathrm{~g} \mathrm{~kg}^{-1}$, e $2,20 \mathrm{~g} \mathrm{~kg}^{-1}$, respectivamente, para essas cultivares. Dentro do sistema convencional, as cultivares diferem entre si com maiores teores para a Prata-Anã e Maravilha (4,06 e 3,90 g kg-1, respectivamente ) e menor teor para a cultivar Thap Maeo $\left(2,43 \mathrm{~g} \mathrm{~kg}^{-1}\right)$.

$\mathrm{O}$ sistema de cultivo influenciou significativamente nos teores foliares de boro (Tabela 8), considerado essencial para a bananeira, obtendose maiores valores em sistema orgânico, exceto para a Pacovan, que não diferiu estatisticamente entre os sistemas. Já no sistema convencional, não houve diferença estatística entre as cultivares dentro do sistema, apresentando teor foliar médio de 17,21 $\mathrm{g} \mathrm{kg}^{-1}$. Contudo, em ambos os sistemas, os valores obtidos estão acima da média proposta por Raij et al. (1997) para a cultivar Pacovan Ken. O micronutriente cobre apresentou diferença estatística, apresentando os maiores teores na cultivar Prata-Anã em sistema orgânico, com valor de $9,00 \mathrm{mg} \mathrm{kg}^{-1}$ e em cultivo convencional apresentou valor de $6,00 \mathrm{mg} \mathrm{kg}^{-1}$; no entanto, não diferiram estatisticamente entre as cultivares no sistema convencional. A mesma cultivar apresentou diferença significativa também em relação ao manganês entre os manejos orgânico (137,33 mg kg-1) e convencional $\left(73,61 \mathrm{mg} \mathrm{kg}^{-1}\right)$. Os micronutrientes zinco e ferro diferiram entre as cultivares dentro do sistema convencional e orgânico, sendo que os teores desses micronutrientes foram superiores no sistema orgânico apenas para a cultivar Thap Maeo. Para o elemento zinco, o menor valor encontrado foi na cultivar Thap Maeo em manejo convencional, com $16,00 \mathrm{mg} \mathrm{kg}^{-1}$, e em sistema convencional com $12,66 \mathrm{mg} \mathrm{kg}^{-1} \mathrm{em}$ sistema orgânico. A mesma cultivar apresentou também menores valores em sistema convencional e orgânico para o elemento ferro, apresentando diferenças significativas das cultivares dentro dos sistemas. (Tabela 8).

Os micronutrientes são os elementos mais encontrados nos restos culturais, que podem ter contribuído para a absorção desse nutriente pelas plantas. Já os teores foliares de zinco e ferro não foram influenciados pelo sistema de cultivo, no entanto, estão acima dos padrões propostos por Raij et al. (1997). Apesar de muitos elementos estudados estarem abaixo da faixa, não foram observados sintomas de deficiência desse nutriente nas plantas em ambos os sistemas de cultivo.

TABELA 1 - Descrição das cultivares de bananeiras (Musa spp.) avaliadas em sistema de cultivos convencional e orgânico.Cruz das Almas - BA.

\begin{tabular}{|c|c|c|}
\hline Cultivar & Grupo & Descrição \\
\hline Caipira & AAA & $\begin{array}{l}\text { Cultivar de porte médio a alto. Internacionalmente conhecida como 'Yangambi Km } \\
5 \text { '. Apresenta resistência às sigatokas amarela e negra e ao mal-do-Panamá. }\end{array}$ \\
\hline Maravilha & $\mathrm{AAAB}$ & $\begin{array}{l}\text { Híbrido resultante do cruzamento entre a 'Prata-Anã' (AAB) e 'SH3142' (AA). } \\
\text { Cultivar de porte médio. Suscetível às sigatokas amarela e negra e ao mal-do-Panamá. }\end{array}$ \\
\hline Prata Anã & $\mathrm{AAB}$ & $\begin{array}{l}\text { Foi selecionada a partir da cultivar Branca. Suscetível às sigatokas amarela, e negra } \\
\text { e ao mal-do-Panamá. Cultivar de porte médio. }\end{array}$ \\
\hline Pacovan Ken & $\mathrm{AAAB}$ & $\begin{array}{l}\text { Híbrido da 'Pacovan' com o diploide 'M53' Resistentes às sigatokas negra e amarela } \\
\text { além do mal-do-panamá. Resistente ao despencamento e apresenta porte alto. }\end{array}$ \\
\hline Thap Maeo & $\mathrm{AAB}$ & $\begin{array}{l}\text { Introduzida da Tailândia e selecionada pela Embrapa Mandioca e Fruticultura. Cultivar } \\
\text { de porte médio a alto. Apresenta frutos arredondados semelhantes à 'Mysore'. }\end{array}$ \\
\hline Tropical & AAAB & $\begin{array}{l}\text { Híbridos tetraploides resultantes da variedade 'Yangambi }{ }^{\circ} 2 \text { ' com o híbrido (AA) } \\
\text { 'M53'. Apresenta porte médio a alto. Apresenta frutos semelhantes ao da cultivar } \\
\text { Maçã. }\end{array}$ \\
\hline
\end{tabular}

Fonte: Silva et al. (2006). 
TABELA 2- Desenvolvimento vegetativo de cultivares melhoradas de bananeira (Musa spp.), no terceiro ciclo de produção, implantadas em sistemas de cultivo convencional e orgânico. Cruz das Almas-BA.

\begin{tabular}{lcccccccc}
\hline \multirow{2}{*}{ Cultivar } & \multicolumn{2}{c}{ APL $(\mathrm{cm})$} & \multicolumn{2}{c}{ DIA $(\mathrm{cm})$} & \multicolumn{2}{c}{ NFVF (dia) } & \multicolumn{2}{c}{ NFVC (dia) } \\
\cline { 2 - 8 } & CON & ORG & CON & ORG & CON & ORG & CON & ORG \\
\hline Caipira & $212,75 \mathrm{~dB}$ & $293,16 \mathrm{dA}$ & $17,79 \mathrm{bA}$ & $17,10 \mathrm{cA}$ & $9,58 \mathrm{bA}$ & $9,57 \mathrm{cA}$ & $4,50 \mathrm{cB}$ & $6,68 \mathrm{aA}$ \\
Maravilha & $279,41 \mathrm{cB}$ & $320,50 \mathrm{cA}$ & $23,91 \mathrm{aA}$ & $24,20 \mathrm{aA}$ & $8,66 \mathrm{bB}$ & $10,58 \mathrm{cA}$ & $5,70 \mathrm{bA}$ & $6,33 \mathrm{aA}$ \\
Pacovan Ken & $366,58 \mathrm{aB}$ & $402,76 \mathrm{aA}$ & $23,62 \mathrm{aA}$ & $23,42 \mathrm{aB}$ & $10,50 \mathrm{aB}$ & $11,84 \mathrm{bA}$ & $5,61 \mathrm{bA}$ & $5,50 \mathrm{bA}$ \\
Prata-Anã & $263,83 \mathrm{cA}$ & $276,92 \mathrm{dA}$ & $21,83 \mathrm{aB}$ & $22,19 \mathrm{bA}$ & $10,75 \mathrm{aA}$ & $10,30 \mathrm{cA}$ & $4,76 \mathrm{cA}$ & $4,41 \mathrm{cA}$ \\
Thap Maeo & $279,41 \mathrm{bB}$ & $381,61 \mathrm{aA}$ & $19,55 \mathrm{bB}$ & $22,01 \mathrm{bA}$ & $9,91 \mathrm{aB}$ & $12,92 \mathrm{aA}$ & $7,61 \mathrm{aA}$ & $7,33 \mathrm{aA}$ \\
Tropical & $309,75 \mathrm{bB}$ & $352,90 \mathrm{bA}$ & $22,45 \mathrm{aB}$ & $24,04 \mathrm{aA}$ & $10,41 \mathrm{aA}$ & $10,72 \mathrm{cA}$ & $5,72 \mathrm{bA}$ & $5,75 \mathrm{bA}$ \\
\hline \multicolumn{1}{c}{ Médias } & 285,33 & 337,80 & 21,55 & 22,16 & 9,96 & 10,98 & 5,65 & 6,00 \\
\multicolumn{1}{c}{ CV $(\%)$} & \multicolumn{2}{c}{10,31} & \multicolumn{2}{c}{11,99} & & 12,56 & & 23,95 \\
\hline
\end{tabular}

${ }^{1}$ Médias seguidas de letras iguais, minúsculas na coluna (Scott Knott) e maiúsculas na linha (Tukey), dentro da mesma característica, não diferem a 5\% de probabilidade. ALT- Altura de planta; DIA - Diâmetro do pseudocaule; NFVF - número de folhas vivas no florescimento; NVFC - número de folhas vivas na colheita. Sistema de manejo: CON - convencional; ORG - orgânico.

TABELA 3- Precocidade de cultivares melhoradas de bananeira (Musa spp.) cultivadas em sistemas convencional e orgânico.Cruz das Almas - BA.

\begin{tabular}{lcccccc}
\hline \multirow{2}{*}{ Cultivar } & \multicolumn{2}{c}{ DPE (dias) } & \multicolumn{2}{c}{ DPC (dias) } & \multicolumn{2}{c}{ DFC (dias) } \\
\cline { 2 - 7 } & CON & ORG & CON & ORG & CON & ORG \\
\hline Caipira & $1.069,08 \mathrm{bA}$ & $1.088,33 \mathrm{bA}$ & $1.200,00 \mathrm{cA}$ & $1.191,58 \mathrm{cA}$ & $107,83 \mathrm{aB}$ & $122,50 \mathrm{aA}$ \\
Maravilha & $1.157,66 \mathrm{aB}$ & $1.255,08 \mathrm{aA}$ & $1.372,41 \mathrm{aA}$ & $1.257,25 \mathrm{bB}$ & $117,33 \mathrm{aA}$ & $98,83 \mathrm{aA}$ \\
Pacovan Ken & $1.154,07 \mathrm{aA}$ & $1.170,33 \mathrm{bA}$ & $1.289,08 \mathrm{bA}$ & $1.256,76 \mathrm{bA}$ & $121,75 \mathrm{aA}$ & $102,69 \mathrm{aB}$ \\
Prata-Anã & $1.149,46 \mathrm{aA}$ & $1.132,25 \mathrm{bA}$ & $1.265,33 \mathrm{bA}$ & $1.269,00 \mathrm{bA}$ & $121,00 \mathrm{aA}$ & $119,53 \mathrm{aA}$ \\
Thap Maeo & $1.113,92 \mathrm{bA}$ & $1.153,38 \mathrm{bA}$ & $1.281,00 \mathrm{bA}$ & $1.205,00 \mathrm{cB}$ & $128,16 \mathrm{aA}$ & $91,07 \mathrm{aB}$ \\
Tropical & $1.213,27 \mathrm{aA}$ & $1.218,91 \mathrm{aA}$ & $1.330,25 \mathrm{aA}$ & $1.328,81 \mathrm{aA}$ & $111,33 \mathrm{aA}$ & $115,54 \mathrm{aA}$ \\
\hline Médias & $1.142,91$ & $1.169,71$ & $1.289,67$ & $1.251,40$ & 117,90 & 128,72 \\
CV(\%) & \multicolumn{2}{c}{7,05} & & \multicolumn{2}{c}{6,06} & \multicolumn{2}{c}{16,24} \\
\hline
\end{tabular}

${ }^{1}$ Médias seguidas de letras iguais, minúsculas na coluna (Scott Knott) e maiúsculas na linha (Tukey), dentro da mesma característica, não diferem a 5\% de probabilidade. DPF - dias do plantio ao florescimento (dias); DPC - dias do plantio à colheita (dias); DFC - dias do florescimento à colheita (dias). Sistema de cultivo: CON: convencional; ORG: orgânico.

TABELA 4- Produtividade de cultivares de bananeira (Musa spp.) no terceiro ciclo de produção em sistemas convencional e orgânico. Cruz das Almas-BA.

\begin{tabular}{lcccccc}
\hline & \multicolumn{2}{c}{ PCA $\left(\mathrm{kg} \mathrm{ha}^{-1}\right)$} & \multicolumn{2}{c}{ PTP $\left(\mathrm{kg} \mathrm{ha}^{-1}\right)$} & \multicolumn{2}{c}{ NFR (un) } \\
\hline \multicolumn{1}{c}{ Cultivar } & CON & ORG & CON & ORG & CON & ORG \\
\hline Caipira & $12.458,33 \mathrm{bA}$ & $13.325,00 \mathrm{bA}$ & $12.300,00 \mathrm{bA}$ & $11.175,00 \mathrm{bA}$ & $156,66 \mathrm{bA}$ & $165,91 \mathrm{bA}$ \\
Maravilha & $19.237,50 \mathrm{aA}$ & $20.366,66 \mathrm{aA}$ & $19.008,33 \mathrm{aA}$ & $19.008,33 \mathrm{aA}$ & $124,16 \mathrm{cA}$ & $107,75 \mathrm{cA}$ \\
Pacovan Ken & $12.908,33 \mathrm{bA}$ & $12.030,76 \mathrm{bA}$ & $10.950,00 \mathrm{bA}$ & $11.100,00 \mathrm{bA}$ & $84,08 \mathrm{dA}$ & $98,50 \mathrm{cA}$ \\
Prata Anã & $12.100,00 \mathrm{bA}$ & $9.476,82 \mathrm{bA}$ & $8.750,00 \mathrm{bA}$ & $8.584,61 \mathrm{bA}$ & $96,33 \mathrm{dA}$ & $108,83 \mathrm{cA}$ \\
Thap Maeo & $16.141,66 \mathrm{aA}$ & $16.253,84 \mathrm{aA}$ & $15.963,63 \mathrm{aA}$ & $15.161,53 \mathrm{aA}$ & $209,63 \mathrm{aA}$ & $212,25 \mathrm{aA}$ \\
Tropical & $12.008,33 \mathrm{bA}$ & $10.927,27 \mathrm{bA}$ & $10.536,36 \mathrm{bA}$ & $10.536,36 \mathrm{bA}$ & $101,09 \mathrm{dA}$ & $108,66 \mathrm{cA}$ \\
\hline Médias & $14.142,35$ & $13.730,05$ & $12.918,05$ & $12.594,30$ & 128,65 & 129,16 \\
CV $\%$ \% & \multicolumn{3}{c}{36,22} \\
\hline \multicolumn{3}{c}{36,46} & \multicolumn{3}{c}{26,28} \\
\hline
\end{tabular}

${ }^{1}$ Médias seguidas de letras iguais, minúsculas na coluna (Scott Knott) e maiúsculas na linha (Tukey), dentro da mesma característica, não diferem a 5\% de probabilidade. PCA - Peso do cacho; PTP - Peso total das pencas; NFR - Número de frutos do cacho. 
TABELA 5- Avaliação de produtividade de cultivares de bananeira (Musa spp.) em sistema de cultivos convencional e orgânico. Cruz das Almas-BA.

\begin{tabular}{lcccccc}
\hline \multirow{2}{*}{ Cultivar } & \multicolumn{2}{c}{ NPE (un) } & \multicolumn{2}{c}{ CMF (cm) } & \multicolumn{2}{c}{ DMF (cm) } \\
\cline { 2 - 7 } & CON & ORG & CON & ORG & CON & ORG \\
\hline Caipira & $9,16 \mathrm{bA}$ & $8,08 \mathrm{cA}$ & $10,00 \mathrm{cA}$ & $11,12 \mathrm{cA}$ & $34,00 \mathrm{bA}$ & $33,87 \mathrm{aA}$ \\
Maravilha & $8,66 \mathrm{cA}$ & $8,00 \mathrm{bA}$ & $19,20 \mathrm{aA}$ & $16,87 \mathrm{aB}$ & $37,62 \mathrm{aA}$ & $35,79 \mathrm{aA}$ \\
Pacovan Ken & $6,50 \mathrm{cA}$ & $6,69 \mathrm{cA}$ & $14,60 \mathrm{bB}$ & $16,19 \mathrm{aA}$ & $33,03 \mathrm{bA}$ & $34,57 \mathrm{aA}$ \\
Prata-Anã & $7,83 \mathrm{cA}$ & $7,23 \mathrm{cA}$ & $9,80 \mathrm{cA}$ & $10,00 \mathrm{cA}$ & $34,26 \mathrm{bA}$ & $32,23 \mathrm{aA}$ \\
Thap Maeo & $10,81 \mathrm{aA}$ & $10,69 \mathrm{aA}$ & $9,70 \mathrm{cB}$ & $11,23 \mathrm{cA}$ & $31,12 \mathrm{bB}$ & $34,13 \mathrm{aA}$ \\
Tropical & $7,08 \mathrm{cA}$ & $6,00 \mathrm{cA}$ & $13,53 \mathrm{bA}$ & $13,50 \mathrm{bA}$ & $34,26 \mathrm{bA}$ & $35,95 \mathrm{aA}$ \\
\hline Médias & 8,38 & 7,89 & 12,80 & 13,15 & 34,04 & 34,42 \\
CV (\%) & \multicolumn{2}{c}{16,06} & \multicolumn{2}{c}{14,44} & & 10,45
\end{tabular}

${ }^{1}$ Médias seguidas de letras iguais, minúsculas na coluna (Scott Knott) e maiúsculas na linha (Tukey), dentro da mesma característica, não diferem a 5\% de probabilidade. NPE - Número de pencas; CMF - comprimento médio do fruto; DMF - Diâmetro médio do fruto. Sistema de cultivo: CON - convencional; ORG - orgânico.

TABELA 6- Despencamento e firmeza de frutos de bananeira (Musa spp.) cultivadas em manejos convencional e orgânico. Cruz das Almas-BA.

\begin{tabular}{lcccccc}
\hline \multirow{2}{*}{ Cultivar } & \multicolumn{2}{c}{ DESP (libras) } & \multicolumn{2}{c}{ FCC (Newton) } & \multicolumn{2}{c}{ FSC (Newton) } \\
\cline { 2 - 7 } & CON & ORG & CON & ORG & CON & ORG \\
\hline Caipira & $7,12 \mathrm{bA}$ & $5,65 \mathrm{aA}$ & $5,80 \mathrm{bA}$ & $5,82 \mathrm{aA}$ & $1,25 \mathrm{bB}$ & $1,52 \mathrm{aA}$ \\
Maravilha & $5,80 \mathrm{bA}$ & $3,60 \mathrm{aA}$ & $8,27 \mathrm{aA}$ & $3,00 \mathrm{aA}$ & $1,47 \mathrm{aA}$ & $0,97 \mathrm{cB}$ \\
Pacovan Ken & $3,97 \mathrm{bA}$ & $3,97 \mathrm{aA}$ & $4,52 \mathrm{cA}$ & $4,52 \mathrm{aA}$ & $1,25 \mathrm{bA}$ & $1,25 \mathrm{bA}$ \\
Prata-Anã & $4,60 \mathrm{bA}$ & $4,62 \mathrm{aA}$ & $3,15 \mathrm{dA}$ & $3,15 \mathrm{aA}$ & $1,52 \mathrm{aA}$ & $1,52 \mathrm{aA}$ \\
Thap Maeo & $13,92 \mathrm{aA}$ & $8,00 \mathrm{aA}$ & $2,67 \mathrm{dA}$ & $3,17 \mathrm{aA}$ & $1,25 \mathrm{bB}$ & $1,42 \mathrm{aA}$ \\
Tropical & $5,12 \mathrm{bA}$ & $5,32 \mathrm{aA}$ & $2,30 \mathrm{dA}$ & $3,37 \mathrm{aA}$ & $1,15 \mathrm{bB}$ & $1,35 \mathrm{bA}$ \\
\hline \multicolumn{1}{c}{ Médias } & 6,75 & 5,19 & 4,45 & 3,83 & 1,14 & 1,33 \\
CV(\%) & \multicolumn{2}{c}{27,98} & \multicolumn{2}{c}{36,43} & \multicolumn{2}{c}{13,47} \\
\hline
\end{tabular}

${ }^{1}$ Médias seguidas de letras iguais, minúsculas na coluna (Scott Knott) e maiúsculas na linha (Tukey),dentro da mesma característica, não diferem a 5\% de probabilidade. Sistema de cultivos: DESP - despencamento do fruto; FCC - firmeza do fruto com a casca; FSC - firmeza do fruto sem casca. Sistema de cultivo: CON: convencional; ORG - orgânico. 


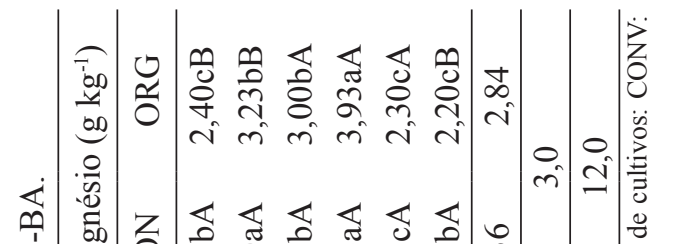

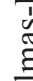

t⿱宀

西

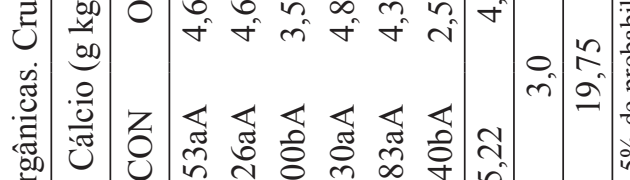

t.

$\frac{0}{a}$

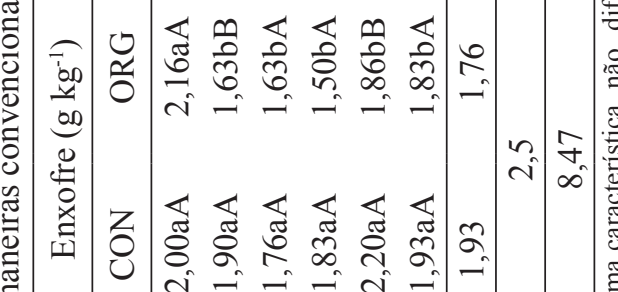

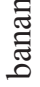

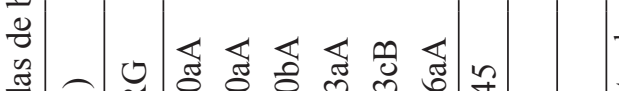

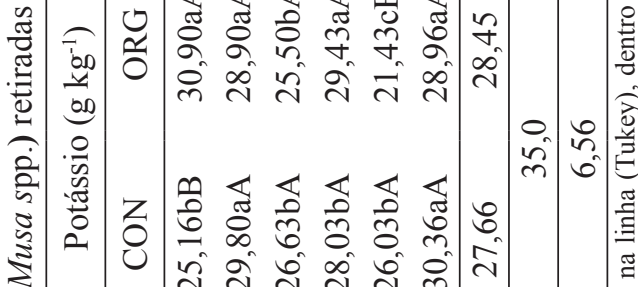

$\underset{3}{2}$

雪

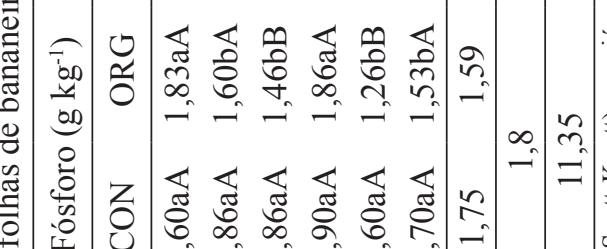

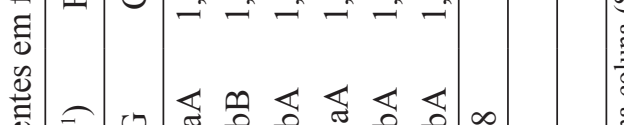

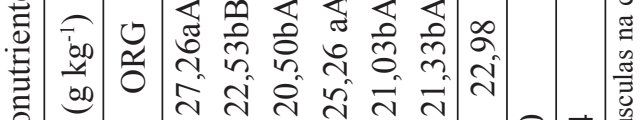

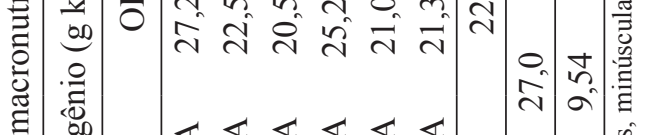

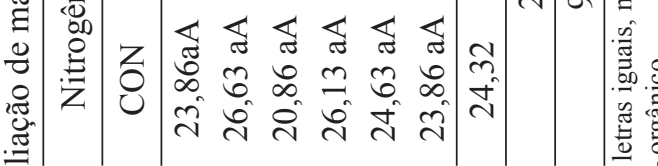

齐 


\section{CONCLUSÕES}

Nas condições em que foi conduzido o trabalho, os resultados obtidos permitem concluir que o sistema de cultivo, orgânico ou convencional, não afeta a produtividade da bananeira. O sistema orgânico proporciona maior desenvolvimento das plantas, especialmente na altura e no diâmetro do pseudocaule, bem como favorece a manutenção de maior número de folhas vivas. O sistema convencional disponibiliza maiores teores de $\mathrm{S}, \mathrm{Mg}, \mathrm{Pe} \mathrm{Ca}$ para as plantas.

\section{AGRADECIMENTOS}

À CAPES (Centro Nacional de Pesquisa de Ensino Superior), pela bolsa de Pós-Graduação, e à Embrapa Mandioca e Fruticultura, pelo incentivo à pesquisa para a realização do trabalho.

\section{REFERÊNCIAS}

AZADI, H.; HO, P. Genetically modified and organic crops in developing countries: a review of options for food security. Biotechnology Advances, New York, v.28, p.160-168, 2010.

DAMATTO Jr. E. R.; VILLAS BOAS, R. L.; LEONEL, S.; FERNANDES, D. M. Alterações em propriedades de solo adubado com doses de composto orgânico sob cultivo de bananeira. Revista Brasileira de Fruticultura, Jaboticabal, v.28, n.3, p.546-549, 2006.

DANTAS, D.J.; MEDEIROS, A.C; NUNES, G.H.S.; MENDOÇA, V.; MOREIRA, M.A.B. Reação de cultivares de bananeira ao Cosmopolites sordidus no Vale do Açu - RN. Revista Verde, Mossoró, v.6, n.3, p.152-155, 2011

GONÇALVES, V.D.; NIETSCHE, S.; PEREIRA, M.C.T.; SILVA, S.O.; SANTOS, M.; OLIVEIRA, J.R.; RANCO, L.R.L.; RUGGIERO, C. Avaliação das cultivares de bananeira Prata-Anã, Thap-Maeo e Caipira em diferentes sistemas de plantio no norte de Minas Gerais. Revista Brasileira de Fruticultura, Jaboticabal, v.30, p.371-376, 2008.
LICHTEMBERG, L.A.; LICHTEMBERG, P. dos S. F. Avanços na bananicultura brasileira, Revista Brasileira de Fruticultura, Jaboticabal, v. 33, n.1 - edição especial, p. 29-36, 2011.

OLIVEIRA, T. K. de; LESSA, L. S.; SILVA, S. O.; OLIVEIRA, J. P. de. Características agronômicas de genótipos de bananeira em três ciclos de produção em Rio Branco, AC. Pesquisa Agropecuária Brasileira, Brasília, v.43, n.8, p.1003-1010, 2008.

RAIJ, B. van; CANTARELLA, H.; QUAGGIO, J.A.; FURLANI, A.M.C. (Ed.). Recomendações de adubação e calagem para o Estado de São Paulo. 2.ed. Campinas: Instituto Agronômico/Fundação IAC, 1997. 285p. (Boletim Técnico, 100).

RAMOS, D.P.; LEONEL, S.; MISCHAN, M.M; DAMATTO JUNIOR, E.R. Avaliação de genótipos de bananeira em Botucatu-SP. Revista Brasileira de Fruticultura, Jaboticabal, v.31, n.4, p.10921101, 2009

SANTOS, S. C.; CARNEIRO, L. C.; SILVEIRA NETO, A. M. da; PANIAGO JÚNIOR. E.; PEIXOTO, C. N. Caracterização morfológica e avaliação de cultivares de bananeira resistentes a sigatoka-negra (Mycosphaerella fijiensis Morelet) no sudoeste goiano. Revista Brasileira de Fruticultura, Jaboticabal, v.28, n.3, p.449-453, 2006.

SILVA, S. de O. e; PIRES, E.T.; PESTANA, R.K.N.; ALVES, J.S.; SILVEIRA, D.C. Avaliação de clones de banana Cavendish. Ciência e Agrotecnologia, Lavras, v.30, p.832-837, 2006.

TERRAZAN, P., VALARINI. P. J. Situação do mercado de produtos orgânicos e as formas de comercialização.Informações Econômicas, São Paulo, v.39, n.11, p.42-47, 2009. 